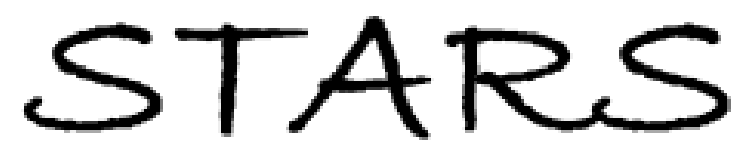

University of Central Florida

STARS

$1-1-2002$

\title{
Reflective reversed-mode polymer stabilized cholesteric texture light switches
}

Hongwen Ren

University of Central Florida

Shin-Tson Wu

University of Central Florida

Find similar works at: https://stars.library.ucf.edu/facultybib2000 University of Central Florida Libraries http://library.ucf.edu

This Article is brought to you for free and open access by the Faculty Bibliography at STARS. It has been accepted for inclusion in Faculty Bibliography 2000 s by an authorized administrator of STARS. For more information, please contact STARS@ucf.edu.

\section{Recommended Citation}

Ren, Hongwen and Wu, Shin-Tson, "Reflective reversed-mode polymer stabilized cholesteric texture light switches" (2002). Faculty Bibliography 2000s. 3424.

https://stars.library.ucf.edu/facultybib2000/3424

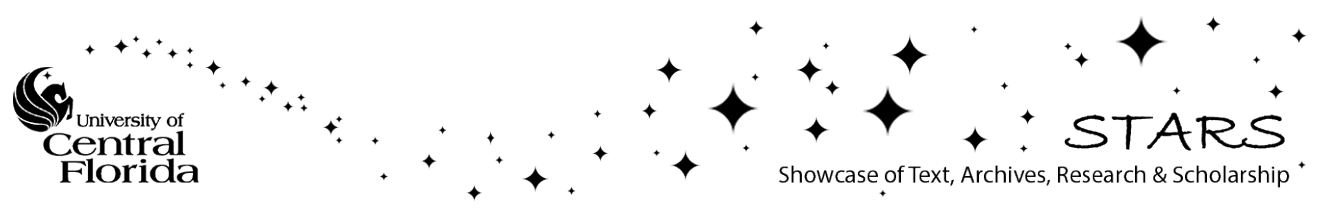




\section{Reflective reversed-mode polymer stabilized cholesteric texture light switches}

Cite as: Journal of Applied Physics 92, 797 (2002); https://doi.org/10.1063/1.1487441

Submitted: 07 January 2002 . Accepted: 23 April 2002 . Published Online: 28 June 2002

Hongwen Ren, and Shin-Tson Wu

\section{ARTICLES YOU MAY BE INTERESTED IN}

Cholesteric liquid crystal/polymer dispersion for haze-free light shutters Applied Physics Letters 60, 3102 (1992); https://doi.org/10.1063/1.106765

Control of reflectivity and bistability in displays using cholesteric liquid crystals Journal of Applied Physics 76, 1331 (1994); https://doi.org/10.1063/1.358518

Two-stage switching behavior of polymer stabilized cholesteric textures Journal of Applied Physics 81, 3007 (1997); https://doi.org/10.1063/1.364335

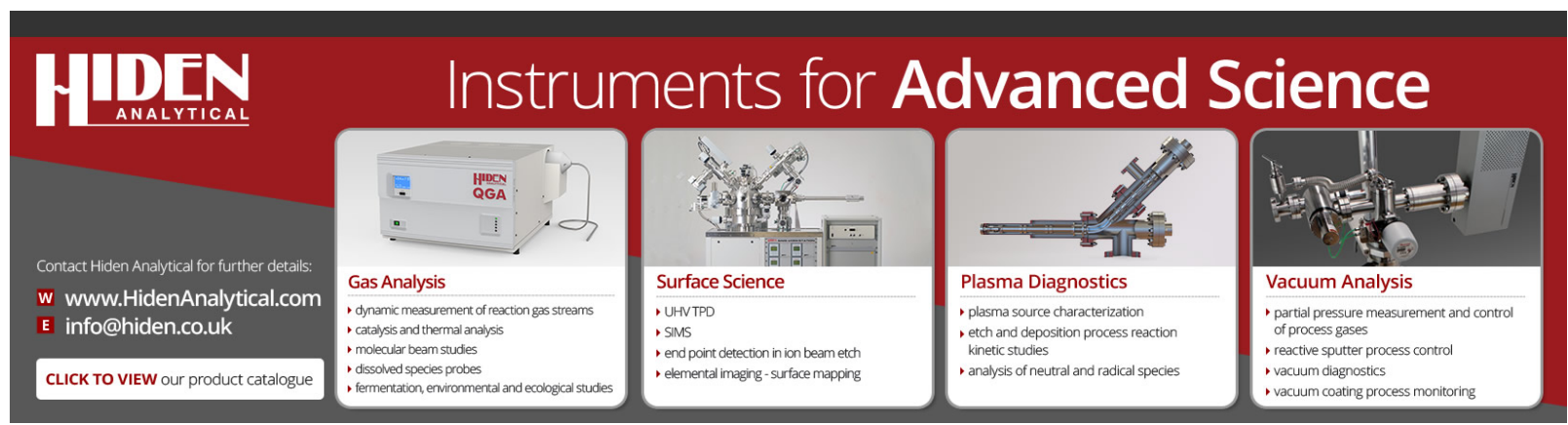




\title{
Reflective reversed-mode polymer stabilized cholesteric texture light switches
}

\author{
Hongwen Ren and Shin-Tson $\mathrm{Wu}^{\mathrm{a})}$ \\ School of Optics/CREOL, University of Central Florida, Orlando, Florida 32816
}

(Received 7 January 2002; accepted for publication 23 April 2002)

\begin{abstract}
Light-switching behavior of reflective reserved-mode polymer-stabilized-cholesteric-texture (PSCT) was analyzed and validated experimentally. Our results indicate that the reflective reversed-mode PSCT offers a much higher contrast ratio and lower operating voltage than its transmissive counterpart. Effects of cell gap, birefringence, and pitch length on the contrast ratios of the reversed-mode PSCT cells were investigated. Potential applications of this device as variable optical attenuator and light shutter for telecommunication are emphasized. (C) 2002 American Institute of Physics. [DOI: 10.1063/1.1487441]
\end{abstract}

\section{INTRODUCTION}

Liquid crystal polymer dispersions with electrically controlled light scattering have been developed for displays and light switches. ${ }^{1-3}$ Based on the morphologies, two types of liquid crystal polymer dispersions have been developed. The first type is the polymer dispersed liquid crystals (PDLC), in which the polymer concentration is around $20 \%-80 \%$ and liquid crystal forms micron-sized droplets dispersed randomly in polymer matrix. Such PDLC film can be switched from a translucent to a transparent state when the applied voltage exceeds a threshold. However, in the transparent state, a strong haze appears at large viewing angles because of the refractive index mismatch between liquid crystal and polymer matrix. The second type is the polymer stabilized cholesteric texture (PSCT), in which the polymer concentration is below $10 \%$ and the polymer in this mixture forms networks for stabilizing the cholesteric texture. In the clear state, PSCT film is free from haze even at a large viewing angle. Thus, it is a strong contender for direct-view displays. ${ }^{4}$

Depending upon the cell fabrication process and substrate surface treatment, a normal-mode (translucent at $V$ $=0$ ) or a reversed-mode (clear at $V=0$ ) PSCT film can be fabricated. Among these two modes, the reversed mode is particularly attractive because it offers an excellent clear voltage-off state, fast switching speed $(<10 \mathrm{~ms})$, large viewing angle $\left(> \pm 60^{\circ}\right)$ and very small hysteresis. ${ }^{5,6} \mathrm{~A}$ shortcoming is its inadequate contrast ratio; increasing cell gap could boost the contrast ratio except that its dark state voltage is also increased proportionally. Another drawback of the thick PSCT cell is that the bulk liquid crystal alignment degrades gradually as the cell gap increases resulting in an undesirable haze in the voltage-off state.

In this article, we have analyzed the light scattering behavior of a reflective reversed-mode PSCT. Our results indicate that the reflective reversed-mode PSCT offers a much higher contrast ratio and lower operating voltage than its transmissive counterpart. Effects of birefringence, pitch length, and cell gap on contrast ratio and operating voltage are studied quantitatively.

\footnotetext{
${ }^{\text {a)} E l e c t r o n i c ~ m a i l: ~ s w u @ m a i l . u c f . e d u ~}$
}

\section{SAMPLE PREPARATION}

To study the birefringence and pitch length effects on the light switching behaviors, we have chosen three LC mixtures: MLC-14600-100 $(\Delta n=0.162)$, E7 $(\Delta n=0.224)$, and BL-038 $(\Delta n=0.272)$, and pitch length $p=3.5$ and $5.0 \mu \mathrm{m}$, respectively. The chiral dopant used is CB15, purchased from Merck. The pitch length $p$ of the PSCT cells is controlled by the concentration of CB15. In our experiments, four PSCT cells including two E7 cells with $p=3.5$ and 5.0 $\mu \mathrm{m}$, one BL-038 cell with $p=3.5 \mu \mathrm{m}$, and one MLC-14600100 cell with $p=3.5 \mu \mathrm{m}$ were prepared.

The concentration of the monomer plays an important role to the performance of the PSCT cells. In our experiments, we found that if the monomer concentration is below $3 \mathrm{wt} \%$, the cholesteric texture cannot be sufficiently stabilized when the electric field is applied. Thus, the voltagedependent transmittance curve is not repeatable after a few cycles. On the other hand, if the concentration is higher than $5-6 \mathrm{wt} \%$, the haze of the cell becomes more apparent. The optimal concentration of the photocurable monomer we employed is around $4 \mathrm{wt} \%$. A small amount of photoinitiator was added to each mixture. The monomer has a common rod-like structure with a reactive double bond at both sides.

The detailed fabrication method of the reversed-mode PSCT cell has been reported in Ref. 4. Each of the above mixtures was sandwiched between two indium-tin-oxide (ITO) coated glass substrates. The inner surfaces of the cell were coated with polyimide and rubbed in antiparallel directions. After the desired alignment of the cholesteric material is achieved, cells are then exposed to UV light to form anisotropic networks. Cell gaps were measured to be $d=5.1$, 10.2, and $15.2 \mu \mathrm{m}$ using a Cary-500 spectrophotometer.

\section{TRANSMISSIVE PSCT}

Figure 1 shows the voltage-dependent transmittance of three reversed-mode E-7 PSCT cells with $p=3.5 \mu \mathrm{m}$ at $\lambda=633 \mathrm{~nm}$. At $V=0$, the cell is highly transparent ( $T$ $\sim 90 \%$ ). As the voltage exceeds a threshold, the transmittance decreases to a plateau due to light scattering of the focal conic state. In a very high voltage regime $\left(>50 \mathrm{~V}_{\mathrm{rms}}\right)$, the cholesteric pitch is completely unwound to a homeotro- 


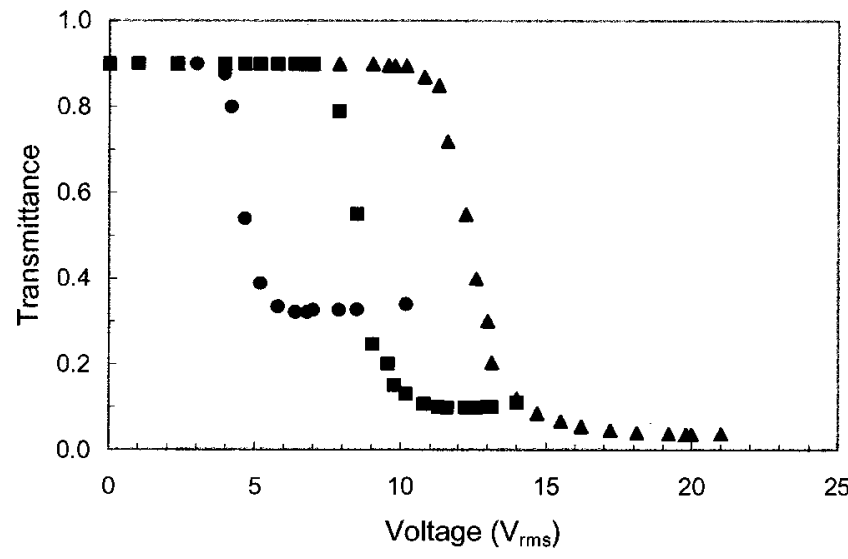

FIG. 1. Measured transmittance of three reversed-mode E7 PSCT cells. Circles: $d=5.1$, squares: $d=10.2$, and triangles: $d=15.2 \mu \mathrm{m}, p=3.5 \mu \mathrm{m}$, and $\lambda=633 \mathrm{~nm}$.

pic state again resulting in a high transmittance. The observed $V_{t h}$ is linearly proportional to the cell gap $d$ as described by de Gennes's theory:

$$
V_{t h}=\frac{\pi^{2} d}{p} \sqrt{\frac{K_{22}}{\Delta \varepsilon}} .
$$

In Eq. (1), the effect of the polymer network is not taken into account. A too high monomer concentration would shrink the domain size and enhance the anchoring strength between the polymer network and bulk liquid crystals. As a result, the threshold voltage is increased. In Fig. 1, all the PSCT cells were prepared at $4 \%$ monomer concentration except for different thicknesses.

Figure 1 shows that the contrast ratio of the 5.1, 10.2, and $15.2 \mu \mathrm{m}$ cells was measured to be $\sim 3: 1,9: 1$, and $25: 1$, respectively. The normalized minimum transmittance $\left(T_{o}\right.$ $=T_{\min } / T_{\max }$ ) follows a simple exponential relationship as:

$$
T_{o}=\exp (-\beta d) \text {, }
$$

where, $\beta$ is the light scattering coefficient at focal conic state and $d$ is the cell gap. As will be shown later, the parameter $\beta$ is mainly determined by the pitch length and LC birefringence. From Eq. (2), the device contrast ratio is simply the inverse of $T_{o}$, i.e., $\mathrm{CR}=\exp (\beta d)$. Increasing cell gap would improve CR exponentially, however, its threshold voltage also increases linearly, accordingly to Eq. (1).

To validate Eq. (2), we plot $-\ln \left(T_{o}\right)$ against cell gap d. A straight line passing through the origin is expected. The slope of the line represents the $\beta$ value. Results are depicted in Fig. 2 for the three LC mixtures studied: MLC-14600-100, E-7, and BL-038 cells at $p=3.5 \mu \mathrm{m}$. Figure 2 shows that the measured slope is $\sim 0.11,0.22$, and $0.24 / \mu \mathrm{m}$ for the MLC14600-100, E-7, and BL-038 cells, respectively. Generally speaking, a higher $\Delta n$ would result in a larger $\beta$. However, this relationship is not so obvious. The slope ratio of E7/ MLC-14600-100 is almost equal to the square of its birefringence ratio. However, the results of BL-038 do not obey this simple relationship. Its slope is only slightly larger than that of E7.

Based on Eq. (2), the contrast ratio is critically influenced by $\beta$ and $d$. The parameter $\beta$ is related to LC birefrin-

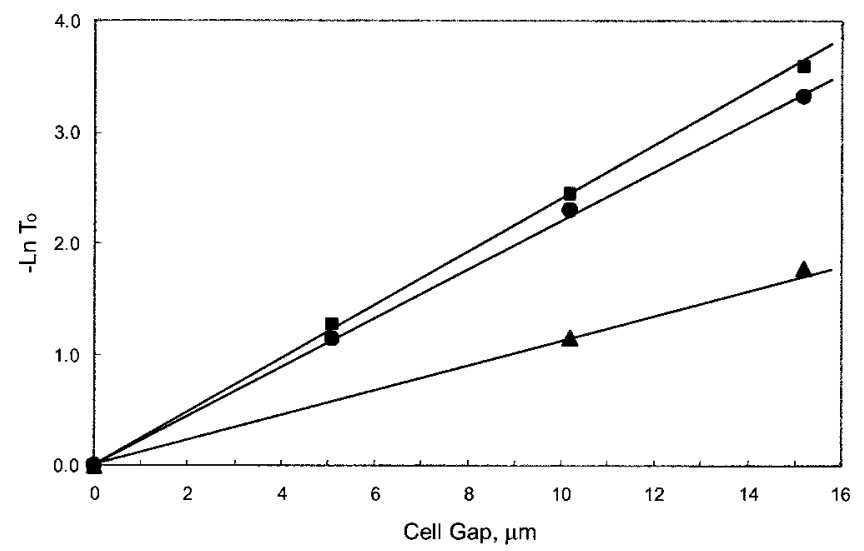

FIG. 2. Plots of $-\ln \left(T_{o}\right)$ vs. cell gap for three transmissive LC PSCT cells. Triangles: MLC-14600-100, circles: E7, and squares: BL-038.

gence and cholesteric pitch length. During our experiments, we found that the PSCT cell with higher birefringence (such as BL-038) exhibits a stronger haze. In order to reduce haze while achieving high contrast ratio, one should choose liquid crystal birefringence in the range of $0.18-0.23$. In addition, the pitch of the cholesteric liquid crystal should satisfy the relationship $p=2 \mathrm{~d} / \mathrm{m}$, where $m$ is a positive integer. When $m=6$ (i.e., $d=3 p$ ), the PSCT cell has optimal electro-optic properties. ${ }^{4}$

We have also examined the pitch length effect on threshold voltage and contrast ratio. Figure 3 compares the results of two transmissive reversed mode E7 PSCT cells with $p$ $=3.5$ and $5.0 \mu \mathrm{m}$. As shown in Eq. (1), the cell with a longer pitch length exhibits a lower threshold voltage. However, its contrast ratio is also lower. As the pitch length gets farther away from the laser wavelength $(p \gg \lambda)$, the light scattering efficiency gradually decreases. Therefore, a large pitch length is favorable for reducing threshold voltage, but unfavorable for contrast ratio.

For telecom light switch applications, a narrow band laser is commonly used. However, to qualify for full-color displays, the PSCT bandwidth must cover the entire visible spectrum. Figure 4 plots the measured transmission spectra $(\lambda=400-800 \mathrm{~nm})$ of an E7 PSCT cell with $p=3.5 \mu \mathrm{m}$ and

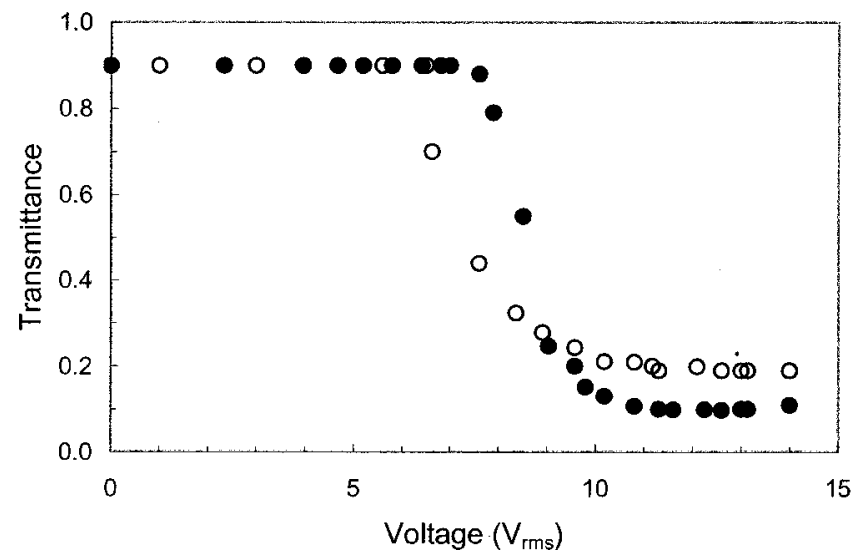

FIG. 3. Voltage-dependent transmittance of two E7 PSCT cells with $p=5$ $\mu \mathrm{m}$ (open circles) and $3.5 \mu \mathrm{m}$ (solids). Cell gap $d=10.2 \mu \mathrm{m}$ and $\lambda=633$ nm. 


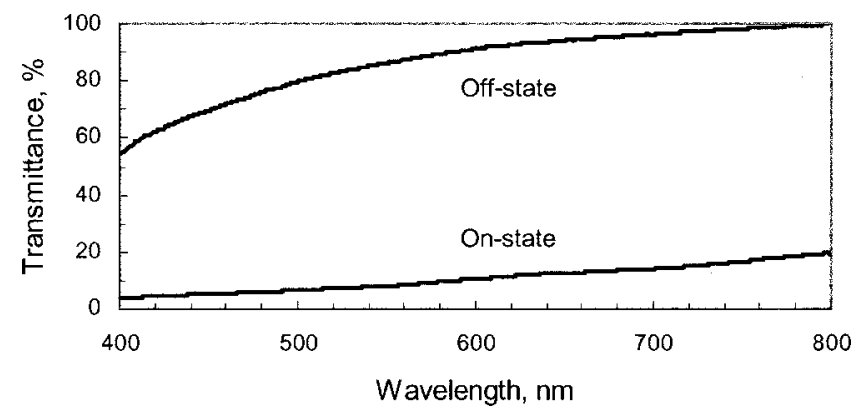

FIG. 4. Wavelength-dependent transmittance of an E7 PSCT cell at voltage-on and off states. $p=3.5 \mu \mathrm{m}, d=10 \mu \mathrm{m}$, and the on-state voltage is $12 \mathrm{~V}_{\mathrm{rms}}$.

$d=10 \mu \mathrm{m}$. In the voltage-off state, the cell is highly transparent in this spectral region. The lower transmission in the blue region results from ITO absorption. In the voltage-on state (with $12 \mathrm{~V}$ applied to the cell), the focal- conic domains scatter light and the cell becomes translucent. As the wavelength increases, the domain sizes become relatively smaller so that their light scattering capability is weakened resulting in an increased leakage. The measured contrast ratio in the visible region remains nearly the same.

\section{REFLECTIVE PSCT}

For a reflective device, the incident light traverses the PSCT layer twice. Thus, the contrast ratio CR has the following form:

$$
\mathrm{CR}=\exp (2 \beta d) \text {. }
$$

The major advantages of the reflective PSCT are twofold: (1) its contrast ratio is square to that of the corresponding transmissive cell, and (2) its dark state voltage is much lower. For example, if a transmissive cell has 10:1 contrast ratio, then the reflective cell should have 100:1 contrast ratio. A 10:1 contrast ratio is inadequate for most transmissive displays. A low contrast ratio implies unsaturated colors. Nevertheless, a 100:1 contrast ratio for reflective direct-view display is quite encouraging. A state-of-the-art reflective direct-

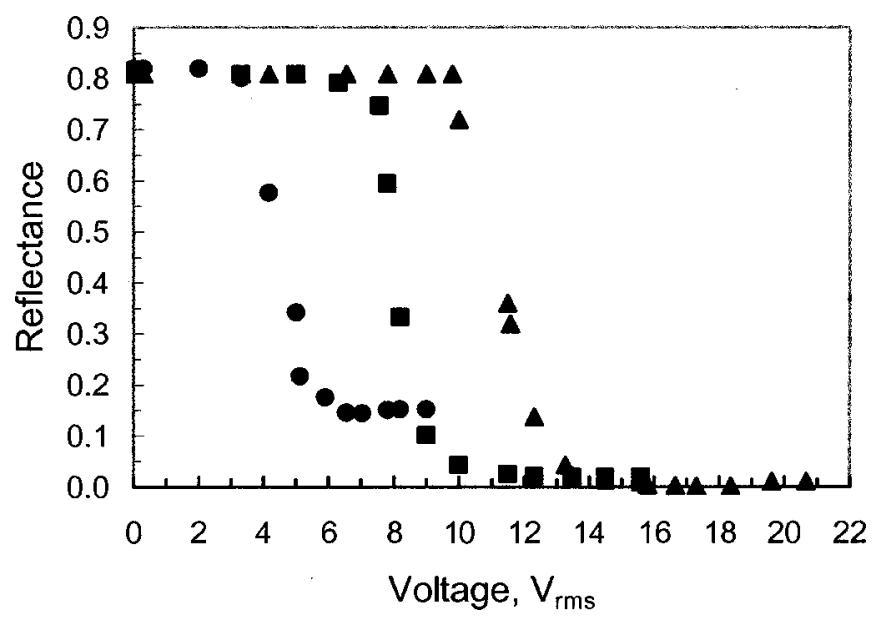

FIG. 5. Measured reflectance of three reversed-mode E7 cholesteric cells. Circles: $d=5.1$, squares: $d=10.2$, and triangles: $d=15.2 \mu \mathrm{m}$. Pitch length $p=3.5 \mu \mathrm{m}, \lambda=633 \mathrm{~nm}$.

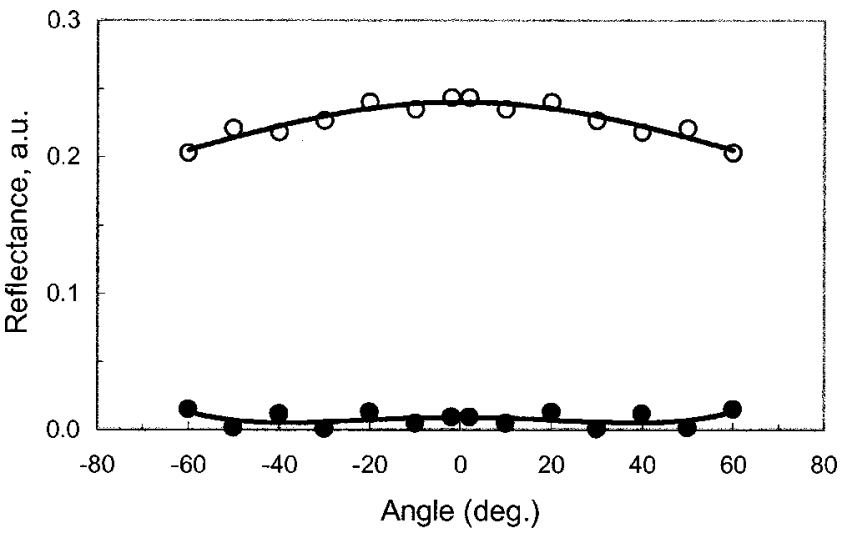

FIG. 6. Viewing angle of a reflective reversed-mode PSCT cell. $d=10 \mu \mathrm{m}$, $p=3.5 \mu \mathrm{m}$, and $\lambda=633 \mathrm{~nm}$. Open circles and solids represent the voltageoff and on state, respectively.

view display has a contrast ratio of $\sim 40: 1$ because of the specular reflection problem. ${ }^{6}$ The threshold voltage of the reflective PSCT remains the same as the transmissive one, however, its dark state voltage is much lower due to the doubled light scattering path.

Figure 5 shows the voltage-dependent reflectance of the three reversed-mode PSCT E7 cells used in Fig. 1. For simplicity, we put a reflector behind the transmissive cell. Here, $100 \%$ represents the reflectance of the mirror alone; multiple reflections due to glass surfaces are neglected. From Fig. 5, the off-state reflectance remains as high as $80 \%$. For the 15 $\mu \mathrm{m}$ cell, a contrast ratio $>700: 1$ is obtained at $V \sim 18 \mathrm{~V}_{\mathrm{rms}}$. Equation (3) was again confirmed experimentally. As expected, the measured slope is twice as large as that of the corresponding transmissive cells.

Wide viewing angle is another important requirement if the reversed-mode PSCT is considered for display applications. Figure 6 plots the polar angle dependent reflectance of a 10- $\mu \mathrm{m}-\mathrm{E} 7$ PSCT cell with $p=3.5 \mu \mathrm{m}$. A HeNe laser was used for such measurements. Because no polarizer is employed, the viewing zone of the reversed-mode PSCT cell is greater than $\pm 60^{\circ}$.

\section{CONCLUSION}

The reflective PSCT offers two major advantages: higher contrast ratio and lower operating voltage, over its transmissive counterpart. Presently, the operating voltage of the reverse-mode PSCT cell is still too high for active matrix displays. However, it is a promising candidate for light switch and variable optical attenuator for fiber-optic telecom application. Since the device is independent of light polarization, it is particularly desirable for those low cost fibers without polarization reservation capability.

\section{ACKNOWLEDGMENTS}

This work was supported by AFOSR under Contract No. F49620-01-1-0377. The authors are indebted to Professor L. Xuan and Professor X. M. Huang of Changchun Institute of Optics, Fine Mechanics and Physics for technical discussions. 
${ }^{1}$ J. L. Fergason, Soc. Inf. Disp., Tech. Dig. 16, 68 (1985).

2 J. W. Doane, N. A. Vaz, B. G. Wu, and S. Zumer, Appl. Phys. Lett. 48, 269 (1986).

${ }^{3}$ A. Y. G. Fuh and O. Caporaletti, J. Appl. Phys. 66, 5278 (1989).

${ }^{4}$ D. K. Yang, L.C. Chien and Y. K. Fung, in Liquid Crystals in Complex Geometries Formed by Polymer and Porous Networks, edited by G. P.
Crawford and S. Zumer (Taylor \& Francis, London, 1996), Chap. 5.

${ }^{5}$ D. K. Yang, L. C. Chien, and J. W. Doane, Appl. Phys. Lett. 60, 3102 (1992).

${ }^{6} \mathrm{~S}$. T. Wu and D. K. Yang, Reflective Liquid Crystal Displays (Wiley, New York, 2001), Chap. 8.

${ }^{7}$ P. G. de Gennes, Solid State Commun. 6, 163 (1968). 